\title{
DEVELOPMENT OF AVIAN INFLUENZA VACCINE ON THE BASIS OF STRUCTURALLY MODIFIED PLANT VIRUS
}

\section{O.A. KONDAKOVA, E.A. TRIFONOVA, M.V. ARKHIPENKO, N.A. NIKITIN, O.V. KARPOVA, J.G. ATABEKOV}

M.V. Lomonosov Moscow State University, Biological Faculty, str. 12, 1, Leninskie gory, Moscow, 119234 Russia, e-mail olgakond1@yandex.ru (corresponding author)

ORCID:

Kondakova O.A. orcid.org/0000-0001-5134-6624

Trifonova E.A. orcid.org/0000-0002-0679-6818

Arkhipenko M.V. orcid.org/ 0000-0002-5575-602X

The authors declare no conflict of interests

Acknowledgements:

The authors thank A.D. Solov'eva and T.V. Gasanova for the assistance in constructing recombinant antigens.

Supported by Russian Science Foundation, a priority program for fundamental research (grant № 14-24-00007)

Received April 27, 2017

\section{Abstract}

Avian influenza is an infectious viral disease that affects various species of birds, including poultry (chicken, turkeys, ducks and geese). Vaccination is a key strategy in the prevention of epizootics and epidemics of influenza. Now, the actual problem is the development of rapid, safe and effective methods for the production of avian flu vaccines. The using of recombinant flu antigen is a promising approach for creating universal, safe and effective poultry flu vaccines. To increasing of low immunogenicity of recombinant antigens plant viruses and virus-like particles can be applied. We have previously shown that spherical particles (SPs) with unique adsorption property and immunostimulating activity. Here we design the candidate vaccine against the avian flu virus (H5N1). The complexes containing the antigenic determinants of the avian flu virus - hemagglutinin (HA) and the extracellular domain of the M2 matrix protein (M2e) presented on the surface of SPs were developed. By indirect immunofluorescence microscopy the specific antigenic activity of recombinant proteins adsorbed on the SPs surface were demonstrated. The SPs-HA-M2e (SPs-HA62/284-M2e) complexes were highly immunogenic. The candidate vaccine induced a strong humoral immune response to both antigenic determinants of avian flu A virus. HA and M2e adsorption on the SPs allowed a 10-fold increase in the production of blood antibodies to antigens in immunized animals. The advantage of this approach for the vaccine development is high efficiency based on the stability and adjuvant activity of SPs, safety and low cost of using plant viruses. Obtaining veterinary vaccines based on structurally modified plant viruses allows the creation of marker veterinary vaccines. Development and creation of marker vaccines is extremely important for the recovery of the poultry population in agro-industrial complexes. The proposed vaccine can be considered as a candidate recombinant vaccine against avian influenza virus.

Keywords: plant viruses, vaccines, spherical particles, influenza virus, recombinant proteins

Flu is one of the most contagious and rapidly spreading infectious diseases [1]. Its causatives are RNA-containing viruses of the family Orthomyxoviridae. Despite the fact that wild birds are a natural reservoir of the flu A virus, these viruses can also infect poultry and several species of mammals, including humans. Epizootic outbreaks, etiologically associated with the flu A virus, cause significant damage to agriculture. At the moment, combatting avian flu involves the introduction of quarantines, as well as the slaughter of infected and contact individuals, which leads to significant economic losses. The greatest danger to humans carried the H5N1 strain of all the flu viruses circulating among birds. Its hazard is due to the high incidence of human infection after the contact with infected birds and the highest rate of deaths [2].

Vaccination is considered to be the most effective way of preventing epidemics and panzootics of flu. In order to obtain modern licensed vaccines 
against flu viruses, researchers use chicken embryos. Production of such vaccines for each strain takes up to 6-9 months, which in the case of the emergence of a new pandemic strain can have unpredictable consequences. Moreover, the use of traditional methods is limited by the high virulence of the H5N1 strain for chicks and chicken embryos, and a potential hazard to humans.

Constructing recombinant proteins is an alternative approach to the creation of universal, safe and efficient vaccines against the avian flu virus [3]. However, a number of studies reported that the viral proteins that are the most promising for inclusion in the vaccine (the surface protein haemagglutinin HA, which serves as the main target for neutralizing antibodies, and the conservative matrix protein M2) have low immunogenicity and cannot stimulate an effective immune response [4-7]. One of the solutions to the problem is using plant viruses [8-10] and virus-like particles to present the determinants of protective antigens and enhance the immune response [11-15].

Earlier, we showed that structural rearrangement of tobacco mosaic virus (TMV) protein after heating spiral virion to $94{ }^{\circ} \mathrm{C}$ led to formation of spherical particles (SPs) of a controlled size. Such SP are biodegradable, they do not contain RNA and have high stability. In addition, SP are safe for humans, since plants and animals do not share common pathogens [16-19]. At the same time, SP are effective immunostimulants [11].

In this work, unique SPs having no analogues were used to create the veterinary candidate vaccine. For the first time, we obtained SP-HA62/284-M2e complexes, containing spherical particles, which were formed during thermal rearrangement of TMV, with recombinant antigens HA62/284 and M2e of the flu A viruses adsorbed, and demonstrated the antigenic specificity of the recombinant proteins. Such complexes appeared to be highly immunodominant: the immunization of laboratory animals shows the production of specific antibodies to both antigenic determinants of avian flu A virus.

The purpose of this study was to develop a new generation of recombinant candidate vaccines against the avian flu virus by assembling in vitro complexes that include immunogenic determinants of HA and M2 proteins of flu A virus on the surface of spherical particles based on the virion of the tobacco mosaic virus.

Techniques. The virions of TMV (strain U1) were isolated from the infected Nicotiana tabacum L. plants of Samsun variety, as it was formerly described [20]. The SPs was obtained from purified TVM preparation $(5 \mathrm{mg} / \mathrm{ml})$ at $94{ }^{\circ} \mathrm{C}$ according to develoeped protocol [18].

Recombinant protein that contains a fragment of the HA molecule of flu A virus $(16 \mathrm{kDa})$ was constructed based on strain A/Kurgan/5/05. The corresponding complementary DNA fragment of the haemagglutinin gene was synthetized in reverse transcription PCR with primers H562-284-P (5'-GCGGATCCGGAGTGAAGCCTCTAATTTTA-AGAGATT-3') and H562-284-M (5'CGTCTAGATTATTCACTTTTCATAATTA-TTGTTAGAGTCCCCT-3'). The amplified fragment was cloned in pQE30 vector (Qiagen N. V., Germany) with BamHI and XbaI restriction sites. The recombinant HA62/284 protein was expressed in Escherichia coli M15 and purified according to the standard procedure [21]. Recombinant $26 \mathrm{kDa}$ protein containing the M2e determinant (23 amino acid residues long) of the flu A virus protein M2, fused to the protein with dehydrofolate reductase, was expressed and purified as described [11]. SP-HA62/284-M2e complexes were prepared in vitro by incubation of HA62/284, M2e and SP proteins in a weight ratio of 5:5:100 at $25{ }^{\circ} \mathrm{C}$ for $20 \mathrm{~min}$.

Sample preparation for electron microscopy was as previously described [22]. The preparations were examined in an electron microscope JEM-1011 
(JEOL, Japan) equipped with a digital camera ES500W Erlangshen (Gatan, Japan). The photomicrographs were analyzed in the ImageJ program (National Institutes of Health, USA).

The antigen specificity of the SP-HA62/284-M2e complex was studied with the use of immunofluorescent microscopy [23]. Rabbit antiserum to HA62/284 protein and mouse antiserum to flu virus M2e protein diluted 1:100 were the primary antibodies. The control samples lack the addition of primary antibodies. The binding of primary antibodies to the complexes was detected with secondary donkey anti-rabbit antibodies conjugated with Alexa Fluor ${ }^{\circledR} 546$ fluorophore (Invitrogen, USA) or secondary chicken anti-mouse antibodies conjugated with Alexa Fluor ${ }^{\circledR} 488$ fluorophore (Invitrogen, USA). The analysis was carried out with the use of Axiovert 200M fluorescence microscope (Carl Zeiss, Germany) equipped with an integrated ORCAII-ERG2 camera (Hamamatsu, Japan).

Immunogenicity of SP-HA62/284-M2e complexes was studied in female white lab mice aged 6-8 weeks with a body weight of 15-18 g divided into 4 groups of 5 animals each. Mice were intraperitoneally (i.p.) immunized with PBS (phosphate-buffered saline, negative control, group 1), free recombinant proteins HA62/284 and M2e (group 2), proteins mixed with Freund's adjuvant (group 3), and SP-HA62 complexes/284-M2e (group 4). The dose per injection was $5 \mu \mathrm{g}$ HA62/284, $5 \mu \mathrm{g}$ of M2e, and $100 \mu \mathrm{g}$ of SP; the volume of the mixture injected was $0.2 \mathrm{ml}$ per animal. A total of 3 immunizations were performed at a 2 -week interval. Blood for analysis was taken 1 week after the last immunization.

The titer of the antisera pool was determined by indirect immunosorbent assay using a Multiscan FC (Thermo Scientific, USA), as described previously [12]. HA62/284 and M2e proteins were used as antigens at a concentration of $10 \mu \mathrm{g} / \mathrm{ml}$. The titer of the antiserum was a dilution in which the optical density of the product of the enzymatic reaction was twice the corresponding value in the negative control (nonimmune mouse serum).

Results. In order to create a candidate vaccine from a purified TMV preparation by means of thermal denaturation at $94{ }^{\circ} \mathrm{C}$, spherical particles were obtained. Transmission electron microscopy was used to control their characteristics (Fig. 1). The size of the obtained SPs was $612 \pm 41 \mathrm{~nm}$.

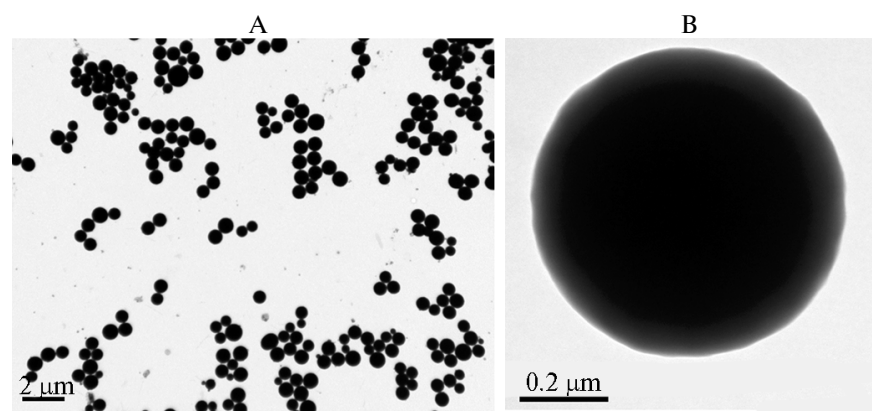

Fig. 1. Electron microphotographs of spherical particles obtained by thermal denaturation of the tobacco mosaic virus. Transmitting electron microscopy (JEM-1011, JEOL, Japan); contrast agent $2 \%$ uranyl acetate; $\times 10,000$ (A) and $\times 200,000$ (B).

The site of the hemagglutinin molecule (amino acid residues 62-284) of strain A/Chicken/Kurgan/05/2005 (H5N1) of influenza A virus was chosen as the antigens that contains the main virus neutralizing epitopes, and the conserved N-terminal extracellular domain of the M2 matrix protein (23 amino acid residues long peptide $\mathrm{M} 2 \mathrm{e}$ ). Hemagglutinin is one of the two major surface proteins of the flu virus. This protein is responsible for the binding the virion to the cell receptors and the fusion of the viral envelope with the cell membrane. HA is the main surface antigen of the flu virus and serves as the main target for neutralizing antibodies. The research showed that the region of the molecule which 

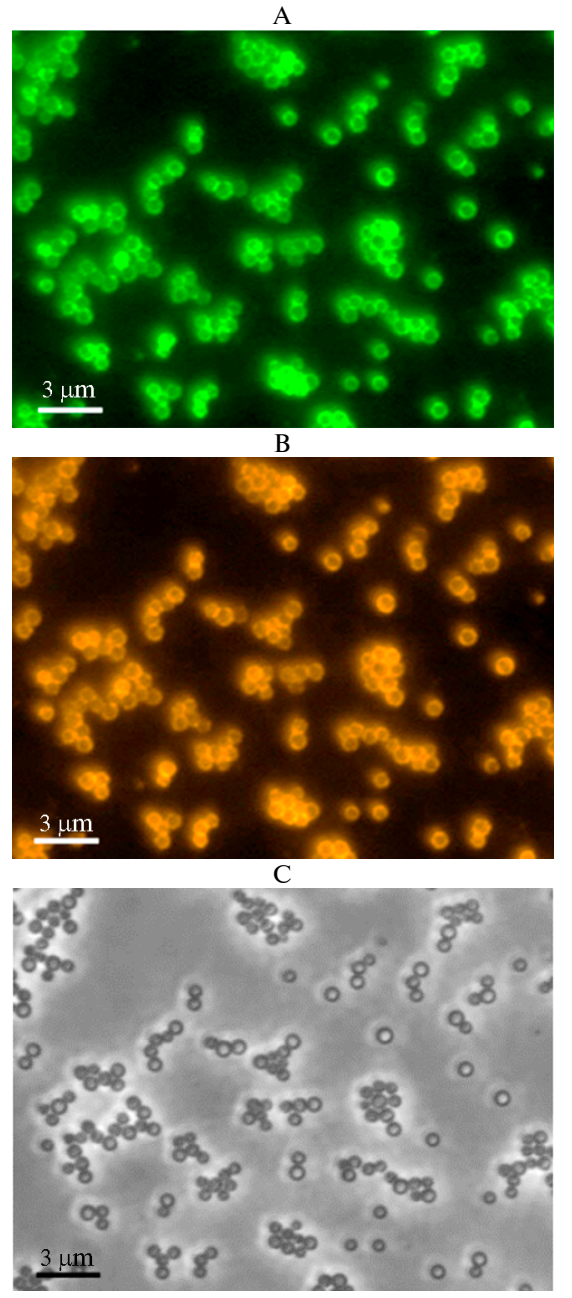

Fig. 2. Immunofluorescent microscopy of the complexes of spherical particles (SPs) obtained by thermal denaturation of tobacco mosaic virus, with the flu virus proteins HA62/284 and M2e simultaneously adsorbed on the SP surface: A - protein M2e detected with primary mouse antibodies and secondary antibodies conjugated with Alexa Fluor $^{\circledR} 488$ fluorophore; B - protein HA62/284 detected in the same sample with primary rabbit antibodies and secondary antibodies conjugated with Alexa Fluor $^{\circledR} 546$ fluorophore; $\mathrm{C}-$ the corresponding image of the sample obtained in the phase contrast mode. Fluorescence microscope Axiovert 200M (Carl Zeiss, Germany). the SP surface. Thus, it has been shown that
the adsorption of HA and M2e proteins on the SP surface does not prevent their binding with specific antibodies to recombinant influenza A viruses.

In assessing the ability of SP complexes with HA and M2e proteins of avian flu A to stimulate a specific immune response, laboratory mice were immunized with free HA62/284 and M2e proteins or with the SP-HA62/284-M2e complex. In the sera of mice immunized with the SP-HA62/284-M2e complex, a significant increase in antibody titer to viral antigens was observed compared to that of free antigens (in case of the SP absence). Thus, when using SPs on the

includs the amino acid residues form 62 to 284 contains most of the neutralizing determinants, as well as the structural elements necessary for efficient folding the recombinant protein [24]. The membrane protein M2 forms ion channels in the virion lipoprotein membrane. An extracellular domain of M2 (M2e), the 22 amino acid residues long fragment of this protein, is exposed on the outer surface of the viral particle. The M2e peptide is evolutionarily conservative and almost identical for all flu viruses circulating in animal populations, including the pandemic viruses. Hence, M2e peptide can be considered as a promising determinant for the development of a universal vaccine against flu [25].

In in vitro obtained SP-HA62/284M2e complexes containing SPs along with the recombinant flu virus antigens HA62/284 and M2e, proteins on the SP surface retained the ability to bind with the specific antibodies to recombinant proteins, as confirmed by indirect immunofluorescence microscopy with two different fluorophores. Fluorescence on the SP surface indicated that both antigens (HA62/284 and M2e) were adsorbed on the same spherical particles and retained antigenic activity in the SP-HA62/284-M2e complexes formed (Fig. 2, A, B). Comparison of the obtained images in the fluorescence (see Fig. 2, A, B) and phase contrast (see Fig. 2, B) modes showed that all the SPs are associated with the molecules of the target protein, while aggregates of antigenic complexes not associated with the SPs, are absent. Therefore, the SPs free of antigen, was not found in the studied preparation. In a negative control, when recombinant proteins or primary antibodies were not used, fluorescence was not observed (data not shown). It indicates the absence of a nonspecific interaction of anti-species antibodies conjugated with the fluorophore with 
surface of which M2e and HA62/284 proteins were simultaneously adsorbed, the titers were $2.0 \times 10^{5}$ for antibodies to M2e protein (Fig. 3, A) and $2.4 \times 10^{5}$ for antibodies to HA62/284 protein (see Fig. 3, B). In the absence of the SPs, in mice immunized with HA62/284 and M2e, the antiserum titer was less than $4.0 \times 10^{4}$. Antiserum titers obtained by immunizing mice with a mixture of HA62/284 and M2e proteins using Freund's adjuvant, one of the strongest immune response stimulants used only in laboratory practice, were $3.0 \times 10^{5}$ and $3.6 \times 10^{5}$, respectively (see Fig. 3, A, B). Thus, the adsorption of HA62/284 and M2e on the SP surface significantly enhanced the immunogenicity of recombinant proteins under intraperitoneal injections resulting in an almost 10-fold increase in the concentration of antibodies to HA62/284 and M2e proteins in the serum of immunized animals compared to that in immunization with free proteins, which is comparable in effectiveness to using Freund's adjuvant.

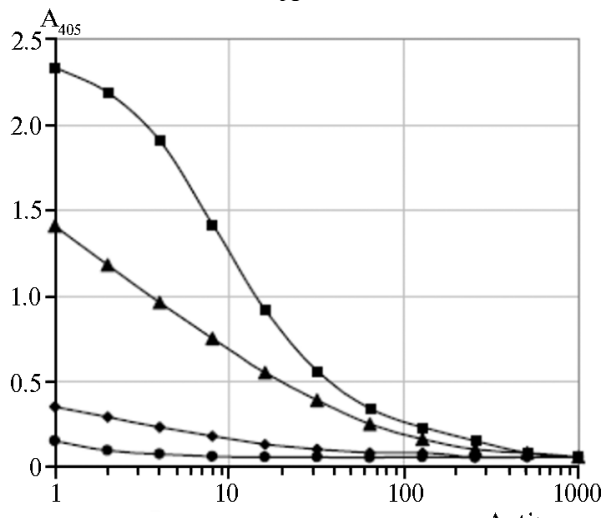

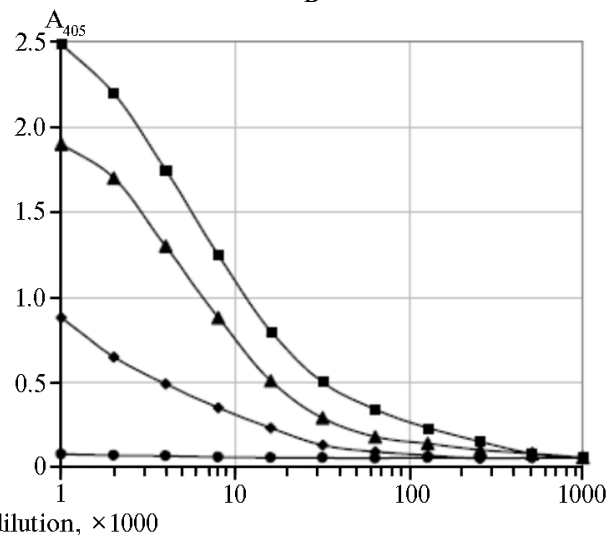

Fig. 3. Adjuvant properties of spherical particles (SPs) obtained by thermal denaturation of tobacco mosaic virus in the candidate vaccine complex with antigenic determinants of avian flu A virus HA62/284 and M2e (SP-HA62/284-M2e) (enzyme immunoassay). The antigens M2e (A) and HA62/284 (B) were immobilized at a concentration of $10 \mu \mathrm{g} / \mathrm{ml}$ on the microplate. The titration curves of antisera during immunization of lab mice with a mixture of free antigens $(\bullet)$, their mixture with addition of Freund's adjuvant (-) and a SP complex (ム) are given. The control was non-immune serum (immunization with phosphate-buffered saline (PBS) solution (•).

It is important to note that the production of veterinary vaccines based on structurally modified plant viruses (SPs) allows the creation of domestic marker veterinary vaccines, i.e. spherical particles, on which a certain part of antibodies is produced during vaccination with the SP pathogen complex [26], can act as a marker which will further distinguish vaccinated birds from carriers of the field virus.

So, the obtained complexes SP-HA62/284-M2e may serve as a basic unit for creating a modern recombinant vaccine against avian flu virus. The advantage of this approach to development of vaccines is their high efficiency, based on the stability and adjuvant activity of spherical particles, as well as the safety and low cost of using plant viruses. The inclusion of the conservative peptide M2e in the developed vaccine preparation should provide protection from both seasonal and probable pandemic viruses. Moreover, it is a marker vaccine that makes it possible to distinguish between vaccinated and unvaccinated individuals that is important for the improvement of commercial poultry populations.

\section{REFERENCES}

1. Nikitin N., Petrova E., Trifonova E., Karpova O. Influenza virus aerosols in the air and their infectiousness. Adv. Virol., 2014, 2014: 1-6 (doi: 10.1155/2014/859090). 
2. Cumulative number of confirmed human cases of avian influenza A(H5N1) reported to WHO. Available http://www.who.int/influenza/human_animal_interface/2017_02_14_tableH5N1.pdf?ua=1. No date.

3. $\mathrm{Hu}$ b e r V.C. Influenza vaccines: from whole virus preparations to recombinant protein technology. Expert Rev. Vaccines, 2014, 13(1): 31-42 (doi: 10.1586/14760584.2014.852476).

4. Treanor J.J., Wilki n s o n B.E., M a s se oud F., Hu-Primmer J., B attaglia R., O'Brien D., Wolff M., Rabinovich G., Blackwelder W., Katz J.M. Safety and immunogenicity of a recombinant hemagglutinin vaccine for $\mathrm{H} 5$ influenza in humans. Vaccine, 2001, 19(13-14): 1732-1737 (doi: 10.1016/S0264-410X(00)00395-9).

5. Nicholson K.G., Colegat e A.E., Podda A., S t e phenson I., Wood J., Y p m a E., Z a mbo n M.C. Safety and antigenicity of non-adjuvanted and MF59adjuvanted influenza A/Duck/Singapore/97 (H5N3) vaccine: a randomised trial of two potential vaccines against H5N1 influenza. Lancet, 2001, 357(9272): 1937-1943 (doi: 10.1016/S01406736(00)05066-2).

6. B l a n c hfie ld K., K a mal R.P., Tzeng W., M u s i c N., Wils on J.R., S t e ve n s J., Li p a to v A.S., K a t z J.M., Y o r k I.A. Recombinant influenza H7 hemagglutinins induce lower neutralizing antibody titers in mice than do seasonal hemagglutinins. Influenza Other Respir. Viruses, 2014, 8(6): 628-635 (doi: 10.1111/irv.12285).

7. Feng J., Zhang M., Mozdzanowska K., Zharikova D., Hoff H., Wu n $\mathrm{n}$ e $\mathrm{r}$ W., $\mathrm{C}$ o u c h R.B., G e $\mathrm{rh}$ a $\mathrm{rd}$ W. Influenza A virus infection engenders a poor antibody response against the ectodomain of matrix protein 2. Virol. J., 2006, 6(3): 102 (doi: 10.1186/1743-422X-3-102).

8. Acosta-Ramírez E., Pérez-Flores R., M aje a u N., P a stelin-Palaci o s R., Gi l-Cruz C., R a mírez-S a ld a ñ a., M a nja r rez-Orduño N., Cervantes-Barragán L., S a ntos - Argumedo L., Flores-Romo L., B e cke r I., I s i b a s i A., L e c le r c D., Ló p e z- M a c í a s C. Translating innate response into long-lasting antibody response by the intrinsic antigen-adjuvant properties of papaya mosaic virus. Immunology, 2008, 124(2): 186-197 (doi: 10.1111/j.1365-2567.2007.02753.x).

9. Li c o C., $\mathrm{C}$ h e $\mathrm{n}$ Q., S a n t i L. Viral vectors for production of recombinant proteins in plants. J. Cell Physiol., 2008, 216(2): 366-377 (doi: 10.1002/jcp.21423).

10. M a nu e 1-Cabrera C.A., Márquez-Aguirre A., Rodolfo H.G., OrtizLazareno P.C., Chavez-Calvillo G., C arrillo-Tripp M., SilvaR o s a l e s L., G u t i é r r e z-O r t e g a A. Immune response to a potyvirus with exposed amino groups available for chemical conjugation. Virol. J., 2012, 9: 75 (doi: 10.1186/1743422X-9-75).

11. Karpova O., Nikitin N., Chirkov S., Trifonova E., Sheveleva A., $\mathrm{L}$ a $\mathrm{z}$ a r e $\mathrm{v}$ a E., A $\mathrm{t}$ a b e $\mathrm{k}$ o $\mathrm{v}$ J. Immunogenic compositions assembled from tobacco mosaic virus-generated spherical particle platform and foreign antigens. J. Gen. Virol., 2012, 93(2): 400-407 (doi: 10.1099/vir.0.036293-0).

12. Trifonova E., Nikitin N., Gmyl A., Lazareva E., Karpova O., Atabekov J. Complexes assembled from TMV-derived spherical particles and entire virions of heterogeneous nature. J. Biomol. Struct. Dyn., 2014, 32(8): 1193-1201 (doi: 10.1080/07391102.2013.816868).

13. L e b e 1 M.E., D a d e 1 i n J.F., C ha rt ra nd K., T a r r a b E., Ka li n k e U., S a vard P., Lab r e c que N., Le c le r c D., L a m a r r e A. Nanoparticle adjuvant sensing by TLR7 enhances CD8+ T cell-mediated protection from Listeria monocytogenes infection. J. Immunol., 2014, 192(3): 1071-1078 (doi: 10.4049/jimmunol.1302030).

14. De n is J., A cost a - R a mirez E., Z h a o Y., H a me 1 in M.E., Kouk avic a I., В a z M., Abed Y., S avard C., Р a й C., Lopez M a cias C., Воivin G., L e c l e r c D. Development of a universal influenza A vaccine based on the M2e peptide fused to the papaya mosaic virus (PapMV) vaccine platform. Vaccine, 2008, 26(27-28): 3395-4003 (doi: 10.1016/j.vaccine.2008.04.052).

15. M a th i e u C., R i o u x G., D u m a s M.C., L e c le r c D. Induction of innate immunity in lungs with virus-like nanoparticles leads to protection against influenza and Streptococcus pneumoniae challenge. Nanomedicine, 2013, 9(7): 839-848 (doi: 10.1016/j.nano.2013.02.009).

16. A t a b e k ov I.G., N i k it i n N.A., K a r p o v O.V. Vestnik Moskovskogo universiteta, Seriya 16. Biologiya, 2015, 4: 29-35 (in Russ.).

17. Nikit in N.A., Trifonova E.A., Karpova O.V., A tabekov I.G. Vestnik Moskovskogo universiteta, Seriya 16. Biologiya, 2016, 3: 20-26 (in Russ.).

18. A t abekov J., Nikitin N., Arkhipenko M., Chirkov S., Karpova O. Thermal transition of native tobacco mosaic virus and RNA-free viral proteins into spherical nanoparticles. J. Gen. Virol., 2011, 92(2): 453-456 (doi: 10.1099/vir.0.024356-0).

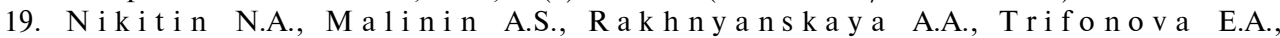
K a r p ov a O.V., Y a r o s lavov A.A., A t a b e k o v J.G. Use of a polycation spacer for noncovalent immobilization of albumin on the thermally modified virus particles. Polym. Sci. Ser. A, 2011, 53(11): 1026-1031 (doi: 10.1134/S0965545X11110083).

20. Trifo nova E.A., Niki t i n N.A., K i r p i c hni k o v M.P., K a r p ova O.V., A t a b e k o v I.G. Vestnik Moskovskogo universiteta, Seriya 16, Biologiya, 2015, 4: 46-50 (in Russ.). 
21. Z a y a kina O.V., Arkhipenko M.V., Kozlovsky S.V., Nikitin N.A., S mi rnov A.A., Sus i P., Rodionova N.P., K a r pova O.V., A t a b e k ov J.G. Mutagenic analysis of Potato Virus X movement protein (TGBp1) and the coat protein (CP): in vitro TGBp1-CP binding and viral RNA translation activation. Mol. Plant Pathol., 2008, 9(1): 37-44 (doi: 10.1111/j.1364-3703.2007.00445.x).

22. Nikitin N., Trifonova E., Evtushenko E., Kirpichnikov M., Atabekov J., $\mathrm{K}$ a r p o v a O. Comparative study of non-enveloped icosahedral viruses size. PLoS ONE, 2015, 10(11): e0142415 (doi: 10.1371/journal.pone.0142415).

23. Nikitin N., Trifonova E., Karpova O., Atabekov J. Examination of biologically active nanocomplexes by nanoparticle tracking analysis. Microsc. Microanal., 2013, 19(4): 808-813 (doi: 10.1017/S1431927613000597).

24. Song L., Naka a r V., Kavita U., Price A., Huleatt J., Tang J., Jacobs A., Liu G., Huang Y., Desai P., Maksymiuk G., Takahashi V., Umlauf S., Reiserova L., Bell R., Li H., Zhang Y., McDonald W.F., Powell T.J., Tuss e y L. Efficacious recombinant influenza vaccines produced by high yield bacterial expression: a solution to global pandemic and seasonal needs. PLoS ONE, 2008, 3(5): e2257 (doi: 10.1371/journal.pone.0002257).

25. Deng L., Cho K.J., Fiers W., Saelens X. M2e-based universal influenza A vaccines. Vaccines, 2015, 3(1): 105-136 (doi: 10.3390/vaccines3010105).

26. Trifonova E., Zenin V., Nikitin N., Yurkova M., Ryabchevskaya E., Put lyaev E., Donchenko E., Kondakova O., Fedorov A., Atabekov J., Karpova O. Study of rubella candidate vaccine based on a structurally modified plant virus. Antiviral Res., 2017, 144: 27-33 (doi: 10.1016/j.antiviral.2017.05.006). 